\title{
Evaluation of oral mucin secretion and pH neutralization by tablet application "Oral pH 7 Rebalancer"
}

\author{
Yun-Ho Choi, Jae young Shin, Brian Chung, Jong Hyun Im, Ji Young Kim, Wonho Ha, Yun-Sun Kim, \\ Nae-Gyu Kang
}

$L G$ Household \& HealthCare, Seoul, Korea

\begin{abstract}
Objective: The aim of this study is to evaluate the efficacy of the chewable LG pH 7 Rebalancer ${ }^{\circledR}$ oral tablet to improve oral environments including maintenance of a neutral oral $\mathrm{pH}$ and promotion of oral mucin secretion in-vivo.

Method: Oral pH and mucin contents were measured before and after LG pH 7 Rebalancer ${ }^{\circledR}$ application. LG pH 7 Rebalancer ${ }^{\circledR}$ contained sodium bicarbonate and tetrasodium pyrophosphate. Alteration in $\mathrm{pH}$ of saliva and recovery of total oral mucin were evaluated by saliva samples which were collected in each time points. In this study, we measured acidity of saliva using $\mathrm{pH}-\mathrm{de}-$ pendent colorimetric assay and total mucin content of saliva using enzyme-linked lectin assay (ELLA). Saliva samples were collected and centrifuged in 13,000 rpm for 10 minutes. Supernatant were collected and reacted with pH indicator in 96-well plate. After 5 minutes of plate shaking, spectral absorbance was measured from 200 to $1000 \mathrm{~nm}$. Individual oral saliva $\mathrm{pH}$ was determined comparing with $\mathrm{pH}$ standards ( $\mathrm{pH}$ 4-pH 10). Oral mucin ELLA assays were performed by using wheat germ agglutinin and HRP-conjugated soybean agglutinin. Total mucin concentrations in saliva samples were determined by comparison with bovine mucin standards.
\end{abstract}

Result: All participants were divided in 3 groups by oral acidity (acidic/ neutral/ basic groups). After LG pH 7 Rebalancer ${ }^{\circledR}$ intake, oral $\mathrm{pH}$ was immediately increased higher to 7.81 comparing to 7.6 of Listerine ${ }^{\circledR}$ Ready! Tabs ${ }^{\mathrm{TM}}$. Unlike Listerine ready tabs ${ }^{\circledR}$ which was totally recovered initial oral $\mathrm{pH}$ (6.86 to 6.84) in 30 minutes, $\mathrm{LG}$ pH 7 Rebalancer ${ }^{\circledR}$ maintained higher $\mathrm{pH}$ (6.82 to 7.12 ) than initial $\mathrm{pH}$ after 30 minutes. Especially, in lower $\mathrm{pH}$ participants who had experienced periodontitis and cavities, neutral oral pH maintaining effects were more long-lasted comparing to Listerine ready tabs. In addition, the total mucin contents were higher in saliva after using LG pH 7 Rebalancer ${ }^{\circledR}$ than Listerine ready tabs ${ }^{\circledR}$. After 30 minutes, LG pH 7 Rebalancer ${ }^{\circledR}$ group showed 1.5 fold higher mucin contents in saliva compared to Listerine ready tabs ${ }^{\circledR}$ groups, which showed lower the level of total mucin compared to saliva before intake. Furthermore, we found the increment of mucin secretion had a negative correlation with the decrement in $\mathrm{pH}$ of saliva. $(\mathrm{R}=-0.81)$.

Conclusion: Dental caries and periodontitis are related to oral environment. In particular, oral saliva plays the important role in maintaining oral health. Our results showed that LG pH 7 Rebalancer ${ }^{\circledR}$ could regulate the acidity of saliva and the secretion of total oral mucin. In conclusion, LG pH 7 Rebalancer $r^{\mathbb{R}}$ could improve oral health through regulating of oral environment.

Keywords: $\mathrm{pH}$ Rebalancer, oral $\mathrm{pH}$, saliva, mucin

Copyright (C) 2021. Korean Academy of Preventive Dentistry. All rights reserved.

This is an Open Access article distributed under the terms of the Creative Commons Attribution Non-Commercial License (http://creativecommons.org/licenses/ by-nc/4.0) which permits unrestricted non-commercial use, distribution, and reproduction in any medium, provided the original work is properly cited. 